\title{
HIGHLY SENSITIVE III-V NITRIDE BASED PIEZORESISTIVE MICROCANTILEVER USING EMBEDDED ALGAN/GAN HFET Abdul Talukdar ${ }^{1 *}$ and Goutam Koley ${ }^{l}$ \\ ${ }^{1}$ University of South Carolina, Columbia, South Carolina, USA
}

\begin{abstract}
In this article, for the first time, a high gauge factor of 3532 is reported using $\mathrm{AlGaN} / \mathrm{GaN}$ Heterostructure Field Effect Transistor (HFET) embedded GaN piezoresistive microcantilever. In addition, deflection transduction signal from the HFET was used to determine dynamic bending (up to $40 \mathrm{~Hz}$ frequency) and $\mathrm{AC}$ response of the cantilever, and also reported for the first time.
\end{abstract}

\section{INTRODUCTION}

Microcantilevers are very attractive for detection of chemicals, explosives, and biological molecules [1-3] due to their high sensitivity and low power consumption. Though optical transduction is widely used and accurate method for transducing mechanical movement of the microcantilever into an electrical signal, piezoresistive and piezoelectric transductions have been demonstrated in order to miniaturize the sensor element. Most of the microcantilever based sensors and their piezoresistive transduction systems are silicon based $[4,5]$, which is not suitable for operating in harsh environments. III-V Nitride semiconductors have attracted wide research interest in optoelectronic and high power microwave devices because of their exceptional physical and chemical properties, which include a wide range of bandgap, high electron mobility, and high breakdown field [6]. Additionally, $\mathrm{GaN}$ related materials exhibit high mechanical and thermal stability coupled with piezoelectric (as well as piezoresistive) properties which make them strong candidates for miniaturized sensors for harsh environments (e.g. automotive, aviation) [7]. The piezoreistive and piezoelectric properties of $\mathrm{AlGaN} / \mathrm{GaN}$ heterostructure is highly attractive for applications in microelectromechanical systems (MEMS), as it can cause large variation in 2-dimensional electron gas (2DEG) at the interface with mechanical strain. The mechanical strain caused by different factors including hydrostatic pressure, biaxial deformation and microcantilever bending [8-10] on 2DEG (and hence the conductivity) and the output characteristics of $\mathrm{AlGaN} / \mathrm{GaN}$ heterostructure field effect transistor (HFET) has already been studied experimentally and theoretically [9-12]. Therefore, $\mathrm{AlGaN} / \mathrm{GaN}$ system can be a good alternative as material and transduction system for developing microcantilever based ultra high detection system. Additionally selective and sensitive detection in a standoff fashion is of great interest at present, with application ranging from homeland security to medical imaging. For example, photoacoustic spectroscopy (PAS) is a standoff method based on detecting acoustic disturbances caused by the infrared absorptions by molecules. PAS can be used to quickly detect very low concentrations. The whole system can be miniaturized to have portable systems capable to in-situ measurements. The photoacoustic system measures absorbed energy and so it is less immune to the background signal in the absence of the sample gas. The sensitivity of the system can be significantly increased by replacing the conventional microphone with a microfabricated cantilever [13]. Previously standard atomic force microscope (AFM) microcantilevers were used as sensitive microscale detectors for photoacoustic signals [14] which seem to be impractical considering AFM setup.

In this article, for the first time, sensing performance of a $\mathrm{GaN}$ piezoresistive microcantilever embedded with $\mathrm{AlGaN} / \mathrm{GaN}$
Heterostructure Field Effect Transistor (HFET) is demonstrated which reports a high gauge factor of 3532. In addition, deflection transduction signal from the HFET was showed for the first time to determine dynamic bending (up to $40 \mathrm{~Hz}$ frequency), AC response of the cantilever, and also acoustic wave resonance which detected vibration amplitude of $22 \mathrm{~nm}$ when the sensor was placed $5 \mathrm{~cm}$ away from an ultrasonic source.

\section{FABRICATION OF MICROCANTILEVER}

A (4" by 4") AlGaN/GaN wafer grown on Silicon (111) substrate was purchased from Nitronex Corporation, USA for this work. The wafer was diced into $32(1.4 \mathrm{~cm}$ by $1.4 \mathrm{~cm})$ pieces. Before dicing, the wafer was spin coated with photo resist (Shipley 1827) and then baked for 5 mins at $110{ }^{\circ} \mathrm{C}$. This is done to avoid contaminating the wafer surface with dust particles generated during dicing. The different layers of the wafer are shown in Fig.1. A $1.1 \mathrm{~m}$ thick layer consisting of $\mathrm{AlN}$ and $\mathrm{AlGaN}$ graded layer was used as a transition layer before growing 1 m undoped $\mathrm{GaN}$ layer. This transition layer along with the undoped $\mathrm{GaN}$ form the thickness of our microcantilevers. On the top of the GaN layer, AlGaN barrier layer of $17.5 \mathrm{~nm}$ thickness was grown (see Fig. 1 for different layers). The Aluminum mole fraction was $x=0.26$. A $2 \mathrm{~nm}$ undoped $\mathrm{GaN}$ cap layer was grown on that. $\mathrm{BCl}_{3} / \mathrm{Cl}_{2}$ based dry etching recipe of $\mathrm{GaN}$ in Inductively Coupled Plasma (ICP) etcher to isolate mesa. $150-200 \mathrm{~nm}$ etch was performed to ensure complete isolation. Same recipe was used for both the AlGaN and $\mathrm{GaN}$ etch. The height was verified by Tencor Profilometer. GaN is etched down in the pocket area up to the substrate where silicon acts as the etch stop layer. Here the etching thickness defines the cantilever thickness. To protect the $2 \mathrm{DEG}$ of the mesa, a $1 \mathrm{~m}$ thick PECVD $\mathrm{SiO}_{2}$ was deposited which was eventually etched away by buffer oxide etch (BOE). Ti(20 nm)/Al(100 nm)/Ti(45 $\mathrm{nm}) / \mathrm{Au}(55 \mathrm{~nm})$ metal stack deposition and rapid thermal annealing $\left(800^{\circ} \mathrm{C}\right.$ for $\left.60 \mathrm{~s}\right)$ were done for ohmic contact formation. For gate contact, Ni $(25 \mathrm{~nm}) / \mathrm{Au}(375 \mathrm{~nm})$ Schottky barrier was used. The GaN cantilever pattern was etched down by inductively coupled plasma (ICP) etch process $\left(\mathrm{Cl}_{2}: 32 \mathrm{sccm}, \mathrm{BCl}_{3}: 8 \mathrm{sccm}\right.$, Ar: 5 sccm; $\mathrm{RF}_{1}$ : $70 \mathrm{~W}, \mathrm{RF}_{2}$ : $\left.500 \mathrm{~W}\right)$. Through wafer Si etching was performed by anisotropic silicon etching from the bottom side (Bosch process). The fabricated microcantilever dimension is $350 \times 50 \times 2 \mu \mathrm{m}$.

\begin{tabular}{|c|}
\hline $20 \AA$ i-GaN \\
\hline $175 \AA$ AlGaN, $26 \%$ Al \\
\hline $1 \mu \mathrm{m}$ i-GaN \\
\hline $\begin{array}{c}1.1 \mu \mathrm{m} \text { AIN/(Al)GaN } \\
(\text { Transition Layer) }\end{array}$ \\
\hline $500 \mu \mathrm{m}$ Si(111) \\
substrate
\end{tabular}

Fig. 1: Different layers of the AlGaN/GaN wafer grown on Si (111) used in this work.

The complete package of the fabricated sample is shown in Fig. 3. 

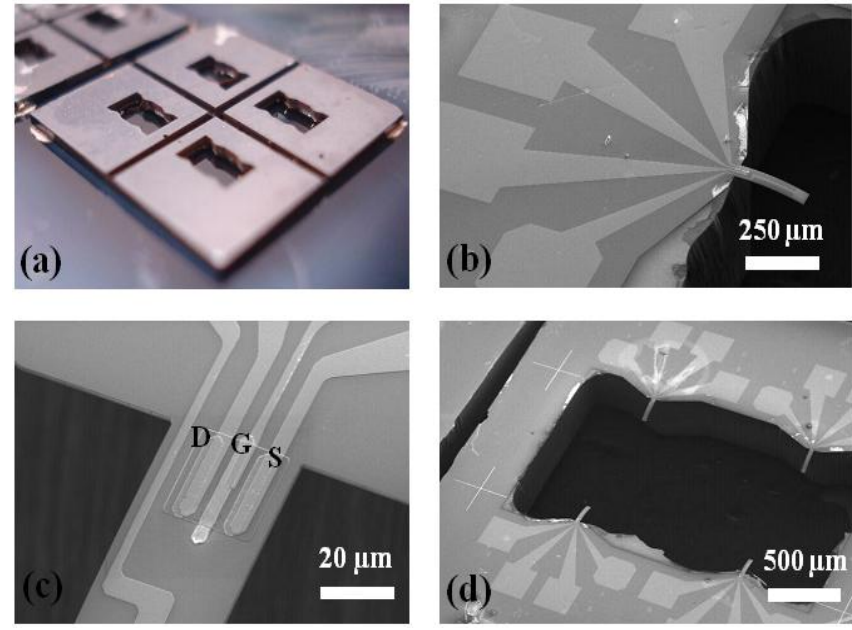

Fig. 2: (a) Image of the sample whic is auto diced into four smaller samples, SEM image of (b) One single microcantilever device with four large bonding pads for drain, gate, source and tip, (c) AlGaN/GaN HFET, (d) a pocket containing four microcantilever devices.

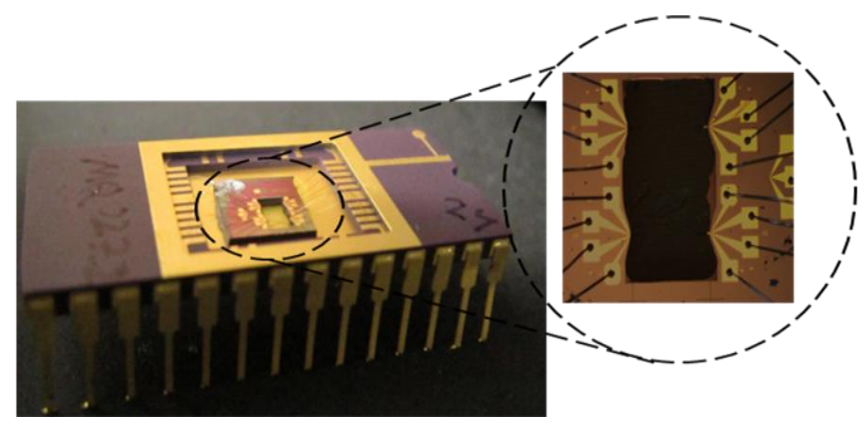

Fig. 3: The complete package of the fabricated sample with wire bonded devices in zoomed view (four microcantilevers).

\section{EXPERIMENTAL SETUP}

The bending experiments on the cantilevers were performed using a Piezo-based Z-axis Nanopositioner purchased from Physik

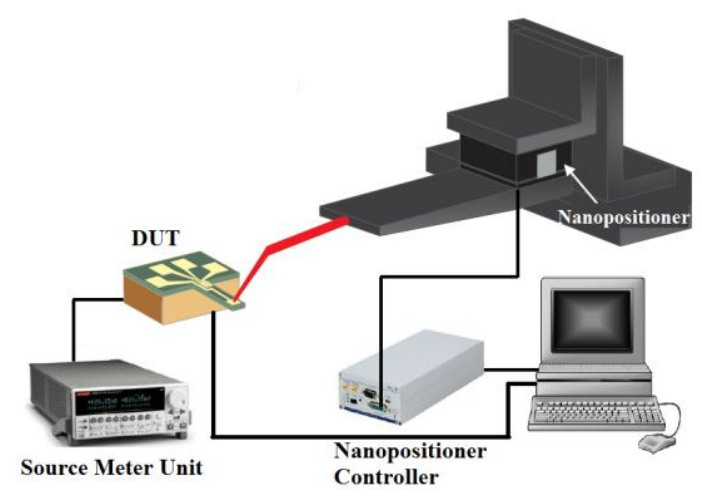

Fig. 4: Schematics of the experimental setup for static and dynamic bending.

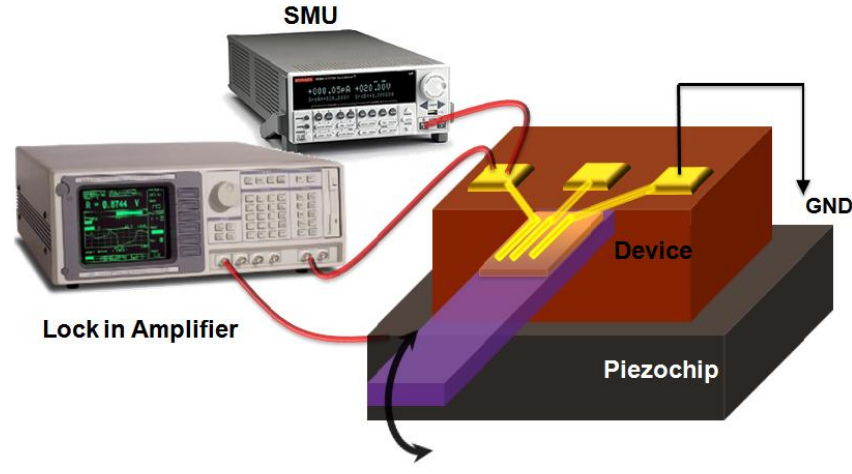

Fig. 5: Schematics of the experimental setup for AC measurement

Instrumente, PI (PI-611 Z) (see Fig. 4). The nanopositioner was attached upside down with a XYZ translation stage (purchased from Thorlabs. Inc.) to allow coarse maneuverability in three axes. The nanopositioner has range of up to $100 \mathrm{~m}$ movement with a closed loop resolution of $2 \mathrm{~nm}$. A micropositioner needle (tip diameter $12 \mathrm{~m}$ ) was attached to the end of the nanopositioner. The nanopositioner is controlled from a computer interface designed in LabView. HFET dc current measurements were performed using Keithley Sourcemeter (2612A). The dc drain current was measured in ambient lighting conditions. We further extended our labview code to perform dynamic bending which helps us to attain low frequency dynamic bending of up to $40 \mathrm{~Hz}$ using the same experimental setup as in Fig. 4. For the AC response of the cantilever a different setup was developed consisting of a miniature peizo actuator (PL055.31 from PI) and a digital lock-in amplifier from Stanford Research Systems (SR850) as shown in Fig. 5. The piezochip has maximum periodic vibration of $2.2 \mathrm{~m}$. Periodic vibration of the pizochip oscillates the cantilver and then the $\mathrm{AC}$ response of the device is extracted by observing the change in HFET current using lock-in amplifier.

\section{RESULTS AND DISCUSSION}

AlGaN/GaN heterostructures has a strain dependent 2dimensional electron gas (2DEG) at the interface, which is sensitive to mechanical load, as well as to chemical modification of the surface, and can be used for novel sensing principles. Both $\mathrm{AlGaN}$ and $\mathrm{GaN}$ will have bending induced polarization at the interface. The $2 \mathrm{DEG}$ carrier density depends on the difference between the polarization of $\mathrm{AlGaN}$ and $\mathrm{GaN}$ at the interface. The whole channel at the $\mathrm{AlGaN} / \mathrm{GaN}$ interface experiences a varying strain along the length. Apart from the change in 2DEG, mobility also gets affected by the change in effective mass. These combined effects of carrier density in 2DEG, and change in mobility offer highly sensitive microsensor with FET embedded GaN microcantilever. The mechanical strain caused by microcantilever bending on 2DEG and the output characteristics of $\mathrm{AlGaN} / \mathrm{GaN}$ HFET have already been studied experimentally [1] and theoretically [2]. At first the DC characteristics of the HFET was recorded and Fig. 6 shows the $I_{d}-V_{d}$ characteristics of one of our best devices for different gate bias. For $\mathrm{AlGaN} / \mathrm{GaN}$ devices, which are normally $\mathrm{ON}$, gate bias can control the channel carrier concentration. The gate modulation helps to reduce the background carrier concentration in 2DEG and thus signifies the deflection induced change of piezoresistivity to increase the sensitivity of such device. We previously reported [15] a gauge factor of $-38\left(\right.$ at $\mathrm{V}_{\mathrm{g}}=0 \mathrm{~V}$ ) and -860 in steady state and transient conditions, respectively. But our second generation devices 


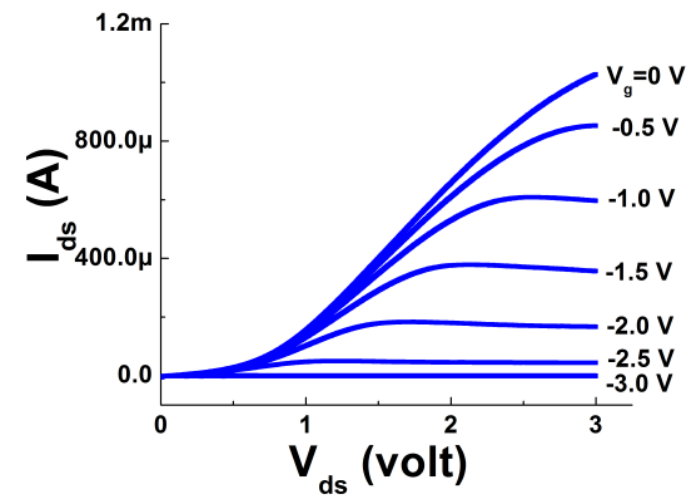

Fig. 6: $I_{d}-V_{d}$ curve for an HEFT device integrated at the cantilever base.

consistently exhibit much higher gauge factor in both static and dynamic bending conditions at zero gate bias. In Fig. 7 the static bending performance of a typical device is shown where the drain current is found change by $1.17 \%$ in magnitude, which gives a gauge factor of 650 . The basic definition of gauge factor is given by: $\mathrm{GF}=(\Delta \mathrm{R} / \mathrm{R}) / \varepsilon$, where $\varepsilon$ is strain, $\Delta R$ is change in resistance, and $R$ is the initial resistance when the cantilever is not bent. The change in drain current is converted to fractional change in resistance of the drain to source contact. Most of our new devices show such higher gauge factor eliminating the need of gate modulation but our best device has exhibited a gauge factor of 3532 which is the highest among its kind ever reported considering zero gate bias to best of our knowledge. We then modified our Labview code for dynamic control of the nanopositioner to bend the microcantilver periodically. Fig. 8 shows the low frequency $(0.5 \mathrm{~Hz})$ response of the same device as in Fig. 7, when the bending magnitude (both downward and upward) was $25 \mathrm{~m}$. We found that the low frequency upward and downward bending does not alter the gauge factor, and the response up to $40 \mathrm{~Hz}$ is also quite similar. Because of the limited response time of the mechanical movement of the nanopositioner, high frequency dynamic bending was not attainable from the setup in Fig. 4. So for extracting the AC response of the devices we used piezochip to attach with the cantilevers as shown in Fig. 5. The piezochip was fed from lock-in amplifier with $1 \mathrm{~V}$ (rms) which generated a periodic vibration on the top surface of the piezochip and thus the cantilever was oscillated. The HFET transduced that oscillation to

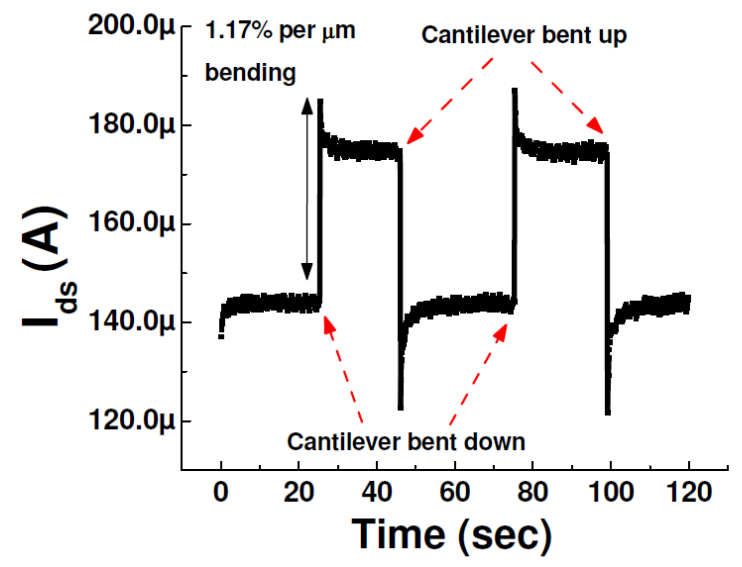

Fig. 7: Static bending response when cantilever is bended (both downward and upward) $25 \mu \mathrm{m}$.

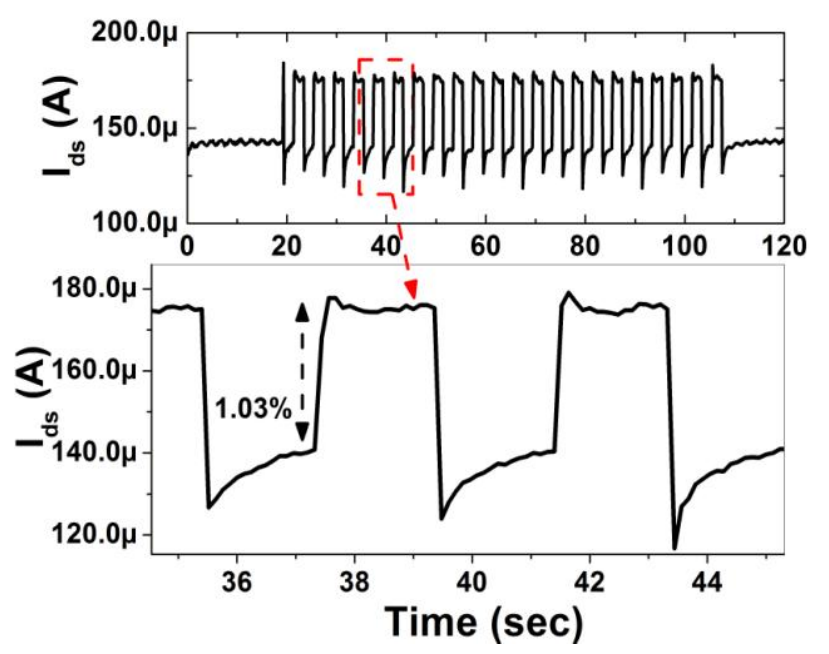

Fig. 8: Static bending response when cantilever is bended (both downward and upward) $25 \mu \mathrm{m}$ and $0.5 \mathrm{~Hz}$ frequency.

resistance change across drain and source which was translated to voltage change in lock-in amplifier. The maximum change in voltage occurred at the resonant frequency of the cantilever which was found $45 \mathrm{KHz}$ (black line graph in Fig. 9). Then the piezochip was held at $1 \mathrm{~cm}$ distance from the device using a micropositioner and was fed again by lock-in amplifier. The periodic vibration of the piezochip generated acoustic wave that propagated by means of adiabatic compression and decompression and eventually oscillated the microcantilever. The resonant peak of the cantilever is found at the same frequency $(45 \mathrm{KHz})$ (blue line graph in Fig. 9). The piezo chip was used as an ultrasonic source and our microcantilever based sensor were able to detect vibrational amplitude of $22 \mathrm{~nm}$ of the ultrasonic generator from $5 \mathrm{~cm}$ distance and $4.6 \mathrm{~nm}$ from $1 \mathrm{~cm}$ distance with a high quality factor of 210 in air. Such HFET transduction setup eliminates the need of conventional laser-photodiode method for acquiring AC response of cantilever. In addition to that, ultrasonic sensing of sub $\mathrm{nm}$ vibration using GaN microcantilever was possible due to the high sensitivity of those sensors.

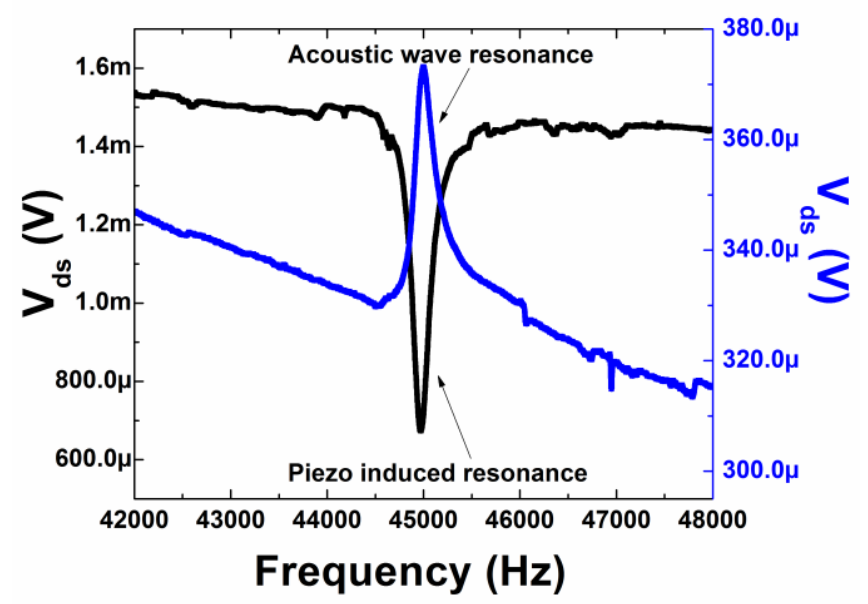

Fig. 9: Resonant peaks for both Piezo induced and acoustic wave induced resonance observed in the $A C$ response of the microcantilevers. 


\section{CONCLUSION}

A high gauge factor of 3532 is reported for the time using GaN piezoresistive microcantilevers embedded with $\mathrm{AlGaN} / \mathrm{GaN}$ HFET without any gate modulation. These sensors have consistent sensitivity in both static and dynamic condition. A novel method of extracting AC response of microcantilevers is also demonstrated. Due to the high sensitivity, those sensors are found efficient as non contact ultrasonic detectors where the preliminary results exhibit the sensing of sub $\mathrm{nm}$ periodic vibration. These results also reveal the capability of using our devices as noncontact ultrasonic remote detector for harsh environment along with sensing different molecular signature using photoacoustic spectroscopy.

\section{ACKNOWLEDGEMENT}

Financial supports for this work from National Science Foundation (Grants Nos. ECCS-0801435 and ECCS-0846898) and Army Research Office (Grant No. W911NF-08-0299) are thankfully acknowledged. We would also like to acknowledge Transducer Research Foundation generously for providing travel support.

\section{REFERENCES}

[1] Z. Y. Hu, T. Thundat, and R. J. Warmack, "Investigation of adsorption and absorption-induced stresses using microcantilever sensors", J. Appl. Phys., 90(1), 427 (2001).

[2] L. A. Pinnaduwage, A. Gehl, D. L. Hedden, G. Muralidharan, T. Thundat, R. T. Lareau, T. Sulchek, L. Manning, B. Rogers, M. Jones, and J. D. Adams, "Explosives: A microsensor for trinitrotoluene vapour", Nature, 425(6957), 474 (2003).

[3] J. Fritz, M. K. Baller, H. P. Lang, H. Rothuizen, P. Vettiger, E. Meyer, H. J. Guntherodt, C. Gerber, and J. K. Gimzewski, "Translating Biomolecular Recognition into Nanomechanics", Science, 288(5464), 316 (2000).

[4] K. Naeli and O. Brand, "Dimensional considerations in achieving large quality factors for resonant silicon cantilevers in air", J. Appl. Phys., 105(1), 014908 (2009).

[5] X. M. Yu, J. Thaysen, O. Hansen, and A. Boisen, "Optimization of sensitivity and noise in piezoresistive cantilevers", J. Appl. Phys., 92(10), 6296 (2002).

[6] H. Morkoc, Handbook of Nitride Semiconductors and Devices, Wiley-VCH, Berlin, 2008.

[7] V. Cimalla, J. Pezoldt, and O. Ambacher, "Group III nitride and $\mathrm{SiC}$ based MEMS and NEMS: materials properties, technology and applications", J. Phys. D: Appl. Phys., 40(20), 6386 (2007).

[8] Y. Liu, P. P. Ruden, J. Xie, H. Morkoc, and K. A. Son, "Effect of hydrostatic pressure on the dc characteristics of $\mathrm{AlGaN} / \mathrm{GaN}$ heterojunction field effect transistors", Appl. Phys. Lett., 88(1), 013505 (2006).

[9] R. Gaska, J. W. Yang, A. D. Bykhovski, M. S. Shur, V. V. Kaminski, and S. M. Soloviov, "The influence of the deformation on the two-dimensional electron gas density in GaN-AlGaN heterostructures", Appl. Phys. Lett., 72(1), 64 (1998).

[10] T. Zimmermann, M. Neuburger, P. Benkart, F. J. HernandezGuillen, C. Pietzka, M. Kunze, I. Daumiller, A. Dadgar, A. Krost, and E. Kohn, "Piezoelectric GaN sensor structures", IEEE Electron Device Lett., 27(5), 309 (2006).

[11] B. Jogai, "Influence of surface states on the two-dimensional electron gas in $\mathrm{AlGaN} / \mathrm{GaN}$ heterojunction field-effect transistors", J. Appl. Phys., 93(3), 1631 (2003).

[12] M. Chu, A. D. Koehler, A. Gupta, T. Nishida, and S. E. Thompson, "Simulation of $\mathrm{AlGaN} / \mathrm{GaN}$ high-electronmobility transistor gauge factor based on two-dimensional electron gas density and electron mobility", J. Appl. Phys., 108(10), 104502 (2010).

[13] N. Ledermann, P. Muralt, J. Baborowski, M. Forster, and J. P. Pellaux, "Piezoelectric $\mathrm{Pb}\left(\mathrm{Zr}_{x}, \mathrm{Ti}_{1-x}\right) \mathrm{O}_{3}$ thin film cantilever and bridge acoustic sensors for miniaturized photoacoustic gas detectors", J. Micromech. Microeng., 14, 1650, (2004).

[14] Brian D. Adamson, John E. Sader, and Evan J. Bieske, "Photoacoustic detection of gases using microcantilevers", J. Appl. Phys., 106, 114510 (2009).

[15] Muhammad Qazi, Nicholas DeRoller, Abdul Talukdar, and Goutam Koley, "III-V Nitride based piezoresistive microcantilever for sensing applications," Appl. Phys. Lett. 99, 193508 (2011)

\section{CONTACT}

*Abdul Talukdar, tel: +1-803-777-3229; talukdaa@email.sc.edu 